\title{
Prevalencia y factores de riesgo de la disfunción sexual femenina: revisión sistemática
}

\author{
Andrea Del Pilar Muñoz D. ${ }^{1, a}$, Paul Anthony Camacho L. ${ }^{2}$ \\ 1,a Estudiante de Pregrado, Programa de Medicina, Facultad de Ciencias de la Salud, Universidad Autónoma de \\ Bucaramanga. ${ }^{2}$ Médico MSc, Facultad de Ciencias de la Salud, Universidad Autónoma de Bucaramanga. Grupo \\ Investigación FOSCAL, Fundación Oftalmológica de Santander. Floridablanca, Santander, Colombia.
}

\section{RESUMEN}

Antecedentes: La disfunción sexual femenina (DSF) se define como un trastorno en el deseo, excitación, orgasmo y/o dolor durante la relación sexual, que genera estrés personal y tiene impacto en la calidad de vida. Objetivo: Analizar datos epidemiológicos sobre prevalencia y factores de riesgo de DSF que permita disponer de un conocimiento unificado sobre estos. Métodos: Se revisaron bases de datos: PubMed y LILACS, entre el 01/01/2004 al 12/10/2014. Se incluyeron estudios de corte transversal que garantizaran los datos de prevalencia y de frecuencia de factores de riesgo. Se evaluó la calidad metodológica utilizando los criterios del STROBE (Strengthening the Reporting of Observational Studies in Epidemiology); se publicaron los resultados en tablas, reportando prevalencia y factores de riesgo con sus respectivos OR. Resultados: Se incluyeron quince artículos, todos cumplieron con más del $70 \%$ de los ítems evaluados por el STROBE. La prevalencia de DSF se encontró entre 5,5 - 73,2\%. El dominio más afectado fue el deseo, con prevalencias entre $10,4 \%-52 \%$. Al combinar los datos las variables asociadas a la presencia de DSF fueron: baja frecuencia de relaciones, edad $>40$ años, bajo nivel educativo, posmenopausia, pareja con disfunción eréctil, entre otros. Conclusión: La DSF posee una alta prevalencia a nivel mundial; la presencia de los múltiples factores de riesgo que presentan las mujeres son motivo de estudio exhaustivo. Un mejor entendimiento de la epidemiologia es vital para planear estrategias de prevención y esquemas de tratamientos efectivos.

\section{PALABRAS CLAVES: Disfunción sexual femenina}

\section{SUMMARY}

Background: Female sexual dysfunction (FSD) is defined as a disorder in desire, arousal, orgasm and / or pain during intercourse, which generates personal stress and impacts the quality of life. Aims: The objective of this review is to analyze epidemiological data on prevalence and risk factors of FSD to provide a unified knowledge. Method: Databases such as PubMed and LILACS were reviewed, from January first of 2004 to October twelve of 2014; cross-sectional studies that ensured information about prevalence and frequency of risk factors were included. Methodological quality was assessed using the criteria of the STROBE (Strengthening the Reporting of Observational Studies in Epidemiology); the results were published in tables, reporting prevalence and risk factors with their respective OR. Results: Fifteen items were included, all met over $70 \%$ of the items evaluated by the STROBE. The prevalence of FSD was found between 5.5 to $73.2 \%$. The most affected domain was the desire with a prevalence between $10.4 \%-52 \%$. The variables associated with the presence of FSD were: low frequency of intercourse, age $>40$ years, low education level, menopause, couples with erectile dysfunction (ED), among others. Conclusion: The FSD has a high 
prevalence worldwide; the multiple risk factors presented by women require further study, because a better understanding of the epidemiology is vital for planning prevention strategies and effective treatment regimens.

\section{KEY WORDS: Female sexual dysfunction}

\section{INTRODUCCIÓN}

La sexualidad es un concepto complejo, consistente en la unión de componentes orgánicos, hormonales y culturales (1) controlados por sistemas intrínsecos del cuerpo humano como el vascular, endocrino y neurológico (2). Los estudios sobre la sexualidad humana se han centrado en su mayoría en la sexualidad masculina y pocos en la femenina, por esta razón el conocimiento sobre la sexualidad femenina se hace necesario, ya que diversos estudios han demostrado que una alteración en el ámbito sexual en las mujeres genera un impacto negativo en su calidad de vida, salud mental y física $(3,4)$.

La disfunción sexual femenina (DSF) se define como un trastorno en el deseo, excitación, orgasmo y/o dolor durante la relación sexual, que genera estrés personal y tiene impacto en la calidad de vida y las relaciones interpersonales (5). Su etiología es multifactorial, debido a sus diversos componentes. Es importante recopilar información sobre la sexualidad femenina, la cual será clave para el diseño de intervenciones en salud sexual (6).

La prevalencia de DSF descrita oscila entre $38 \%-64 \%(2,7)$; dentro de los factores asociados DSF están la edad y la menopausia; las mujeres postmenopáusicas reportan una frecuencia alta de disfunciones sexuales, aun mayor que la de los hombres de la misma edad (8). Al comparar mujeres de 30 años con mujeres de 60 años, aumenta significativamente la prevalencia de síntomas de trastorno orgásmico $(15,2 \%$ a $32,2 \%)$, deseo sexual $(27,7 \%$ a $57,9 \%)$, trastorno de excitación $(29,7 \%$ a $57,9 \%)$ y lubricación $(12,5 \%$ a $51,2 \%)$. Otros factores de riesgo encontrados en la literatura son fumar, estado marital, nivel de educación, religión, trabajo, raza, enfermedad preexistente y depresión $(2,7)$.

El propósito de esta revisión fue analizar datos epidemiológicos sobre prevalencia y factores de riesgo de DSF que permita disponer de un conocimiento unificado sobre estos.

\section{MATERIALES Y MÉTODOS}

Estrategia de búsqueda: Se realizó una revisión bibliográfica en PUBMED y LILACS entre el 1 enero 2004 al 12 de octubre de 2014, para identificar posibles estudios relacionados sobre prevalencia y la presencia de factores asociados de DSF. La búsqueda se hizo combinando términos MeSH (Medical Subject Heading) y texto libre como: "survey", "cross-sectional", "Cross sectional", "transversal", "sexual dysfunction", "risk factors", "prevalence", combinados con términos booleanos. También se realizó búsqueda de fuentes secundarias de los artículos identificados en la búsqueda primaria. Los dos autores independientemente revisaron todos los resúmenes de las citaciones identificadas por la estrategia de búsqueda.

Criterios de elegibilidad: Se incluyeron estudios de corte transversal que garantizaran la disponibilidad de datos de prevalencia y la frecuencia de factores de riesgo de disfunción sexual femenina que hubieran sido publicados entre 01/01/2004 y $12 / 10 / 2014$. Los estudios excluidos fueron todos aquellos en donde la población a estudio era mujeres en estado de gestación, con enfermedades crónicas, hospitalizadas, con consumo crónico de medicamentos e infértiles.

Selección de los estudios y extracción de datos: Los dos autores independientemente aplicaron los criterios de elegibilidad para evaluar las referencias de los artículos obtenidos por la estrategia de búsqueda propuesta, revisando el título y el resumen. Posteriormente, se obtuvieron los artículos a texto completo de todos los artículos que al menos uno de los autores considero potencialmente elegibles. Estos artículos seleccionados fueron analizados por los mismos autores, decidiendo la inclusión de estos en la revisión. Los mismos autores realizaron la extracción de datos de interés y evaluaron la calidad metodológica de los estudios incluidos. Los desacuerdos fueron discutidos y resueltos por consenso.

Calidad metodológica: Se evaluó la calidad metodológica utilizando los criterios del STROBE (Strengthening the Reporting of Observational studies in Epidemiology) (9), el cual consta de 22 ítems a evaluar en cada uno de los artículos seleccionados. Se elaboró una lista de chequeo para evaluar el cumplimiento de los ítems, calculando el porcentaje de ítems cumplidos para cada artículo. Se dividió el nivel de evidencia en tres: A para artículos que cumplieran más del $80 \%$ de los ítems de la lista, B artículos que cumplieran entre $70 \%$ y $80 \%$ de los ítems de la lista, C artículos que cumplieran $<70 \%$. 


\section{RESULTADOS}

Selección de estudios: La búsqueda bibliográfica en PUBMED y LILACS proporcionó un total de 20 artículos que cumplían con los criterios de selección definidos en la estrategia de búsqueda. Tras completar la primera evaluación de títulos y resúmenes se excluyeron 3 artículos por ser estudios realizados en mujeres con enfermedad crónica, 1 por ser en mujeres infértiles, 1 por evaluar conocimiento sobre sexualidad. Los 15 artículos restantes se obtuvieron a texto completo para proceder con la evaluación secundaria, los cuales demostraron cumplir con los requerimientos de la revisión y fueron incluidos en el análisis (Figura 1). La prevalencia de disfunción sexual femenina se encontró entre $5,5-73,2 \%$ (Tabla I).
Características de los estudios: Todos los artículos incluidos correspondían a estudios observacionales, descriptivo de corte transversal tipo encuesta. La búsqueda se inició desde 2004 hasta 2014, los 15 artículos fueron publicados a partir de 2007. El rango de edad de las participantes de los estudios se encontró entre 15 y 70 años. Seis estudios fueron realizados en mujeres casadas y un estudio en mujeres que trabajaban en el área de la salud, siendo este grupo donde se encuentra la prevalencia más baja de disfunción sexual. En 8 artículos se evaluó la prevalencia de disfunción sexual con el Índice de Función Sexual Femenina (FSFI) (10), 4 con la versión validada para su país y 4 con la versión estándar; 14 artículos definían el criterio por el cual consideraban disfunción sexual y solo el de Hayes 2008 no presenta esta información (11) (Tabla I).

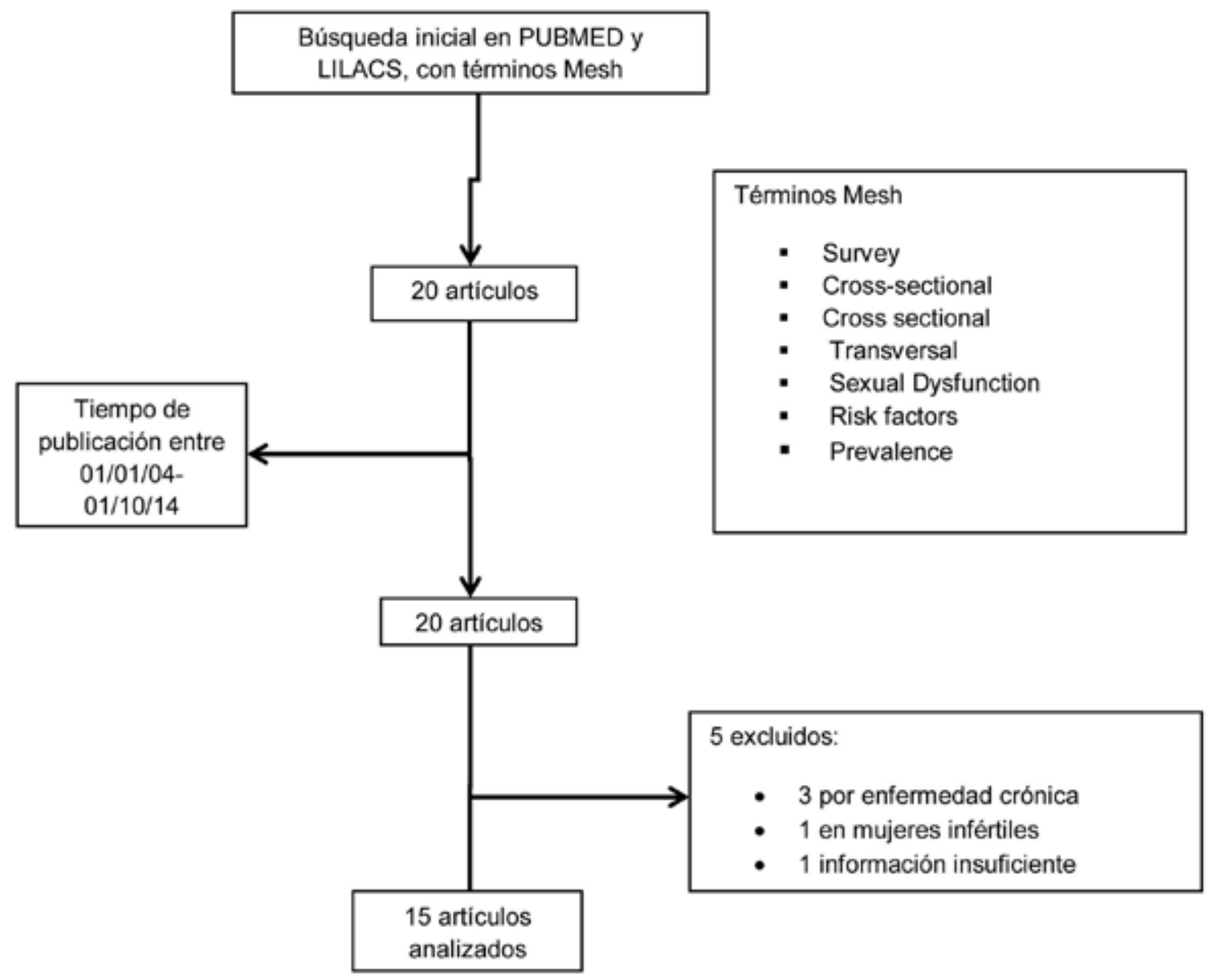

Figura 1. Proceso de selección de los artículos incluidos en el estudio. 


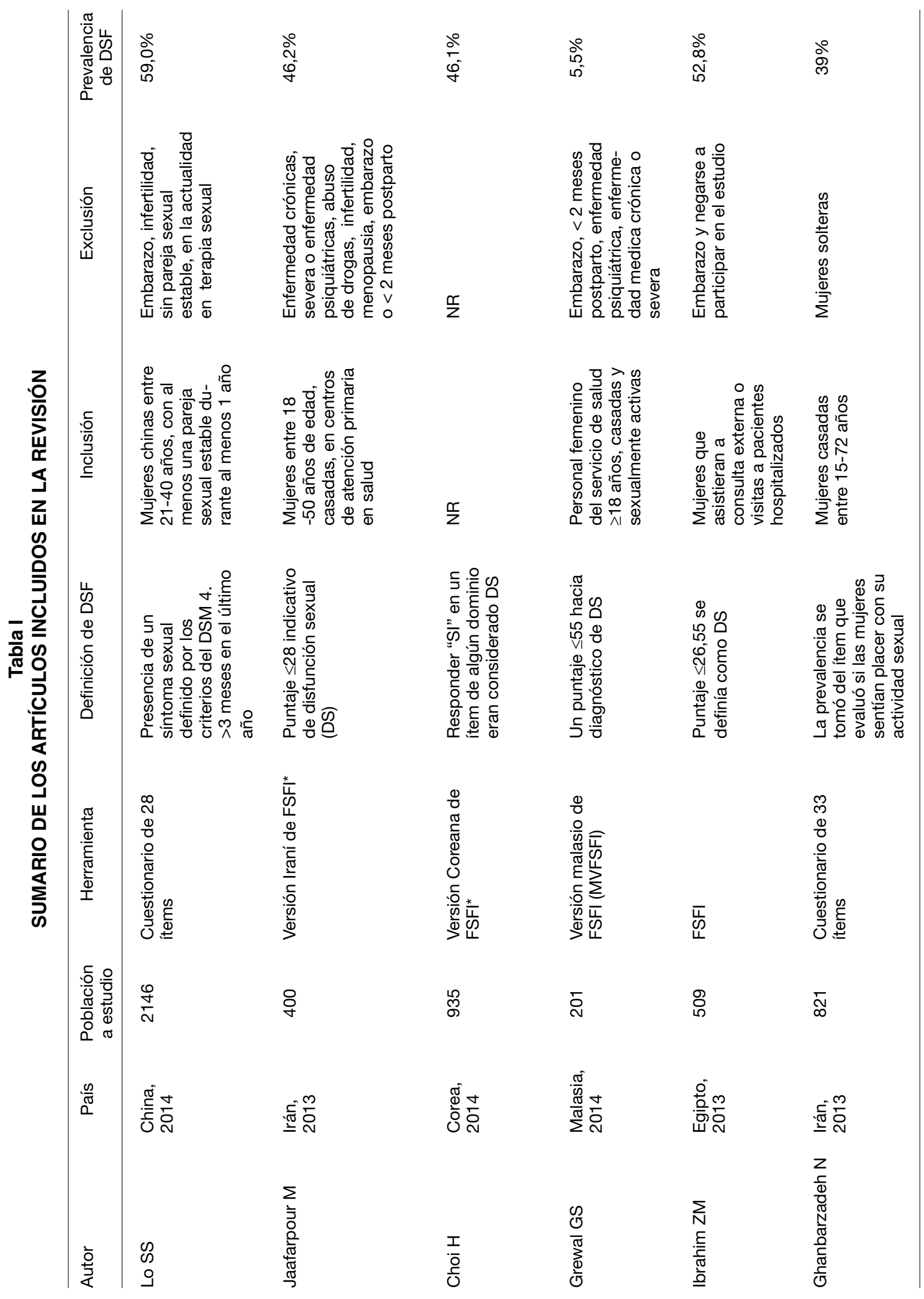




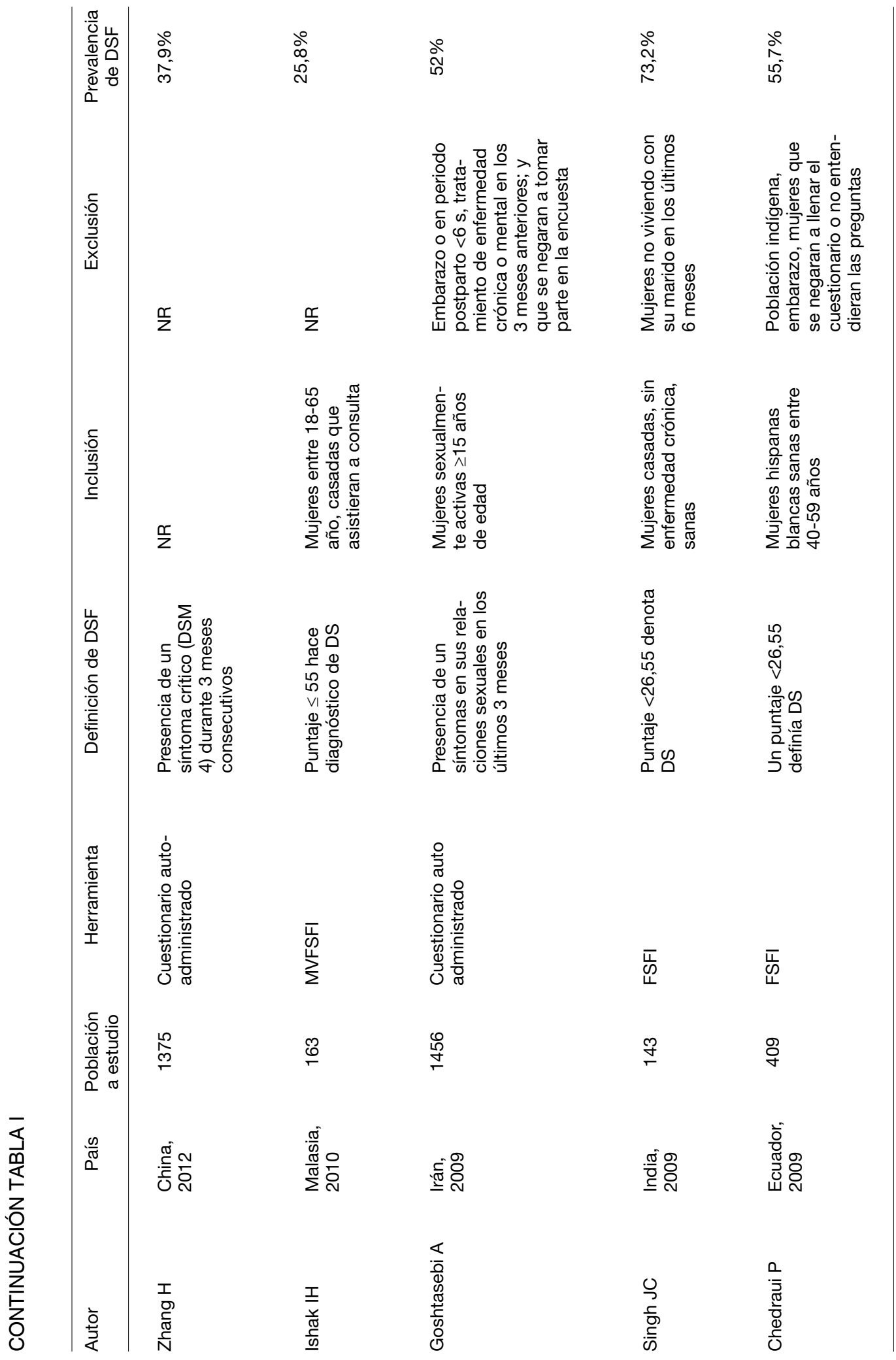




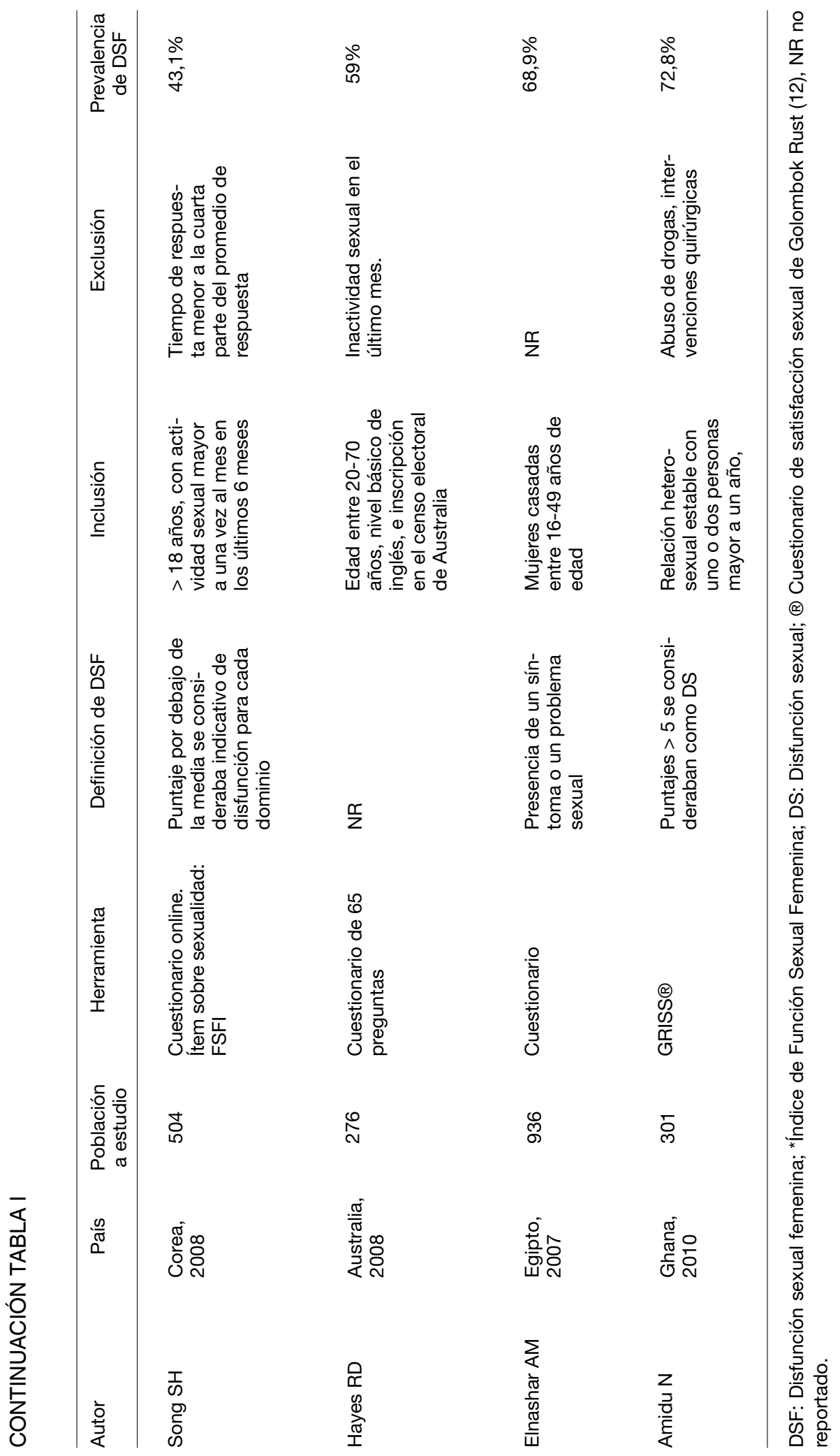


Calidad metodológica: Al aplicar el STROBE a los 15 artículos incluidos en la revisión, 11 cumplieron con más del $80 \%$ de los ítems evaluados, por lo cual se le otorgó una valoración de calidad $A$; de estos 11 artículos, 2 cumplieron con más del 90\% de los ítems evaluados. Cuatro de los artículos incluidos cumplieron entre el $70 \%$ y el $80 \%$ de los ítems del STROBE por lo cual se les otorgó valoración de calidad B (Tabla II).

Prevalencia de tipos de disfunción sexual: 8 artículos evaluaron los 6 dominios completos de la función sexual en sus respectivos estudios, de los cuales 7 utilizaron el FSFI (10), y uno el de Goshtasebi 2009 (13) que utilizó un cuestionario auto administrado. Choi en 2014 utilizo la versión coreana de FSFI; sin embargo, no se puede extraer del artículo la prevalencia de disfunción en el dominio de excitación. En el artículo de Hayes en 2008 (11), las prevalencias se presentan en tablas divididas por grupos de edad, por lo anterior se toma el rango de presentación de estas prevalencias (Tabla III).

La prevalencia de disfunción sexual por dominio se distribuyó: deseo 10,4\% - 52\%, excitación $3,5 \%-91,3 \%$, lubricación 2,5\% - 96,6\%, orgasmo $2 \%-82,6 \%$, dolor $3 \%-64,4 \%$, satisfacción 3,5\% $81,2 \%$. En el estudio de Grewal (14), se encontró las prevalencias más bajas en todos los dominios excepto en el dominio de deseo; las prevalencias más altas de todos los dominios se presentan en el estudio de Singh (15) (Tabla II), estos dos estudios aportan la prevalencia más baja y más alta, respectivamente, de disfunción sexual femenina general (Tabla I).

\section{Tabla II \\ DESCRIPCIÓNY CRITERIOS DE CALIDAD DE LOS ESTUDIOS INCLUIDOS EN LA REVISIÓN}

\begin{tabular}{lcc}
\hline Autor (año) & $\begin{array}{c}\text { Puntaje de } \\
\text { STROBE (\%) }\end{array}$ & $\begin{array}{c}\text { Calidad de la } \\
\text { valoración }\end{array}$ \\
\hline Lo SS (2014) & 81 & $\mathrm{~A}$ \\
Song SH (2008) & 91 & $\mathrm{~A}$ \\
Singh JC (2009) & 86 & $\mathrm{~A}$ \\
Ishak IH (2010) & 81 & $\mathrm{~A}$ \\
Ibrahim ZM (2013) & 81 & $\mathrm{~A}$ \\
Hayes RD (2008) & 95 & $\mathrm{~A}$ \\
Grewal GS (2014) & 86 & $\mathrm{~A}$ \\
Goshtasebi A (2009) & 81 & $\mathrm{~A}$ \\
Ghanbarzadeh N (2013) & 86 & $\mathrm{~A}$ \\
Chedraui P (2009) & 86 & $\mathrm{~A}$ \\
Amidu N (2010) & 81 & $\mathrm{~A}$ \\
Zhang H (2012) & 77 & $\mathrm{~B}$ \\
Jaafarpour M (2013) & 72 & $\mathrm{~B}$ \\
Elnashar AM (2007) & 77 & $\mathrm{~B}$ \\
Choi H (2014) & 77 & $\mathrm{~B}$ \\
\hline
\end{tabular}

El dominio que presento mayor prevalencia de disfunción entre los estudios fue el de deseo, seguido del dominio de orgasmo (Tabla III).

Factores de riesgo de disfunción sexual: En los estudios incluidos en esta revisión, cada uno evaluó diferentes factores de riesgo, los más frecuentes fueron: la baja frecuencia de relaciones sexuales, este demostró ser un factor de riesgo significativo en varios de los estudios incluidos $(16,17)$; con un OR 10,67 (IC95\% 3,58-31,74) (Tabla IV) en el estudio de Ishak y cols (18); pero en el estudio de Grewal y cols (14), no se encontró asociación entre éste y la presencia de disfunción sexual femenina OR 6,74 (IC95\% 0,66-68,54).

La presencia de enfermedad médica de base $(6$, $11,18)$, la presencia de bajo nivel educativo (6), tener solo educación primaria $(16)$ y secundaria $(13,15)$, demostraron ser un factor de riesgo significativo para presentar DS, mientras que ésta no se encontró asociación con las personas que habían recibido algún tipo de educación adicional (11) (Tabla V).

Tener mayor edad representa un factor de riesgo para presentar una DS, la mayoría de estudios que toman como corte para la edad 40 años, mostraron la existencia de asociación significativa entre tener mayor edad y presentar una DS $(1,16,18,19,20)$, exceptuando el estudio de Hayes y cols (11) en el cual esta variable no es significativa OR 0,4 (0,1-1,9) (Tabla IV). En el estudio de Song y cols (17), se observó que tener entre 20-29 años representa un factor de riesgo de presentar una disfunción sexual (OR 3,49; IC95\% 1,48-8,41).

Tener un mayor número de partos no demostró ser una variable significativa en todos los estudios que se evaluó; sin embargo, Elnashar y cols (21), encontraron que presentar más de 5 partos representa un factor protector (OR 0,26; IC95\% 0,16$0,43)$ para presentar una DS (Tabla III).

Tener un matrimonio $\geq 10$ años se asocia a la presentación de DS como se encontró en los estudio de Jaafarpour y cols (16) (OR 1,76; IC95\% 1,04-1,97) y de Ibrahim y cols (19) (OR 2,0; IC95\% $1,2-4,3)$, pero con un matrimonio $\geq 20$ años no se encontró diferencias como lo expresa Grewal y cols (14) (OR 2,49; IC95\% 0,43-14,32) (Tabla V).

Estar desempleada o ser ama de casa se asoció a la presencia de DS según el estudio realizado por Jaafarpour y cols OR 1.34 (IC 95\%1.06-1.63), pero según Ghanbarzadeh y cols (23) y Zhang y cols (22), esta variable no es significativa. En el estudio realizado por Choi y cols (1), la presencia de una actitud negativa por parte de la mujer hacia el sexo, no constituye una variable significativa para presentar una DS (OR 1,26; IC95\% 0,780-2,062), en caso contrario, Song y cols (17) identificaron que el factor de riesgo más fuerte para el desarrollo de una DS es la actitud negativa de la mujer hacia el sexo (OR 19,06; IC95\% 5,67-64,06). 
Tabla III

PREVALENCIA DE ALTERACIÓN DE LOS DOMINIOS DE LA FUNCIÓN SEXUAL FEMENINA

\begin{tabular}{|c|c|c|c|c|c|c|}
\hline Autor (año) & Deseo & Excitación & Lubricación & Orgasmo & Dolor & Satisfacción \\
\hline Lo SS (2014) & $31,8 \%$ & $31,7 \%$ & NR & $40 \%$ & $33,8 \%$ & NR \\
\hline Jaafarpour M (2013) & $45,3 \%$ & $37,5 \%$ & $41,2 \%$ & $42,0 \%$ & $42,5 \%$ & $44,5 \%$ \\
\hline Choi H (2014) & $10,4 \%$ & NR & $42 \%$ & $45 \%$ & $41,1 \%$ & $45,9 \%$ \\
\hline Grewal GS (2014) & $18,9 \%$ & $3,5 \%$ & $2,5 \%$ & $2 \%$ & $3 \%$ & $3,5 \%$ \\
\hline Ibrahim ZM (2013) & $45,5 \%$ & $38,6 \%$ & $39,1 \%$ & $49,2 \%$ & $40,2 \%$ & $41,5 \%$ \\
\hline GhanbarzadeHN (2013) & $15,4 \%$ & NR & NR & $10,5 \%$ & $24,1 \%$ & $26,4 \%$ \\
\hline Zhang H (2012) & $11,7 \%$ & NR & $16,3 \%$ & $14,7 \%$ & $13,8 \%$ & $15,5 \%$ \\
\hline Ishak IH (2010) & $39,3 \%$ & $25,8 \%$ & $21,5 \%$ & $16,6 \%$ & $16,6 \%$ & $21,5 \%$ \\
\hline Goshtasebi A (2009) & $19,3 \%$ & $18,6 \%$ & $11,9 \%$ & $21,3 \%$ & $18,2 \%$ & $19,4 \%$ \\
\hline Singh JC (2009) & $77,2 \%$ & $91,3 \%$ & $96,6 \%$ & $86,6 \%$ & $64,4 \%$ & $81,2 \%$ \\
\hline Chedraui P (2009) & $37,9 \%$ & $48,2 \%$ & $49,4 \%$ & $48,2 \%$ & $49,6 \%$ & $47,4 \%$ \\
\hline Song SH (2008) & $44 \%$ & $49 \%$ & $37,7 \%$ & $32 \%$ & $34,6 \%$ & $37 \%$ \\
\hline Hayes RD (2008) & $22-52 \%$ * & $27-71 \%$ * & NR & $18-52 \%{ }^{*}$ & NR & NR \\
\hline Elnashar AM (2007) & $49,6 \%$ & $36 \%$ & NR & $43 \%$ & $31,5 \%$ & $36,2 \%$ \\
\hline Amidu N (2010) & NR & NR & NR & $72,4 \%$ & $61,8 \%$ & $77,7 \%$ \\
\hline
\end{tabular}

*Rango de presentación del tipo de disfunción, dividido en artículo por grupo de edad. NR: No reportado

Tabla IV

FACTORES DE RIESGO RELACIONADOS SIGNIFICATIVAMENTE CON DISFUNCIÓN SEXUAL FEMENINA

\begin{tabular}{|c|c|c|c|c|c|c|c|c|}
\hline \multirow[t]{2}{*}{ Factores de riesgo } & \multicolumn{8}{|c|}{ Artículos seleccionados y estimadores del efecto utilizado } \\
\hline & $\begin{array}{c}\text { Lo SS } \\
\text { OR }\end{array}$ & $\begin{array}{c}\text { Grewal GS } \\
\text { OR }\end{array}$ & $\begin{array}{c}\text { Ghanbarzadeh N } \\
\text { OR }\end{array}$ & $\begin{array}{c}\text { Zhang } \mathrm{H} \\
\text { OR }\end{array}$ & $\begin{array}{c}\text { Ishak IH } \\
\text { OR }\end{array}$ & $\begin{array}{l}\text { Singh JC } \\
\text { OR }\end{array}$ & $\begin{array}{c}\text { Elnashar AM } \\
\text { OR }\end{array}$ & $\begin{array}{c}\text { Amidu R } \\
\text { OR }\end{array}$ \\
\hline $\begin{array}{l}\text { Casadas o en convi- } \\
\text { vencia }\end{array}$ & 0,55 & & & & & & & \\
\hline $\begin{array}{l}\text { Relaciones sexuales } \leq \\
2 \text { veces a la semana }\end{array}$ & & 5,0 & & & 10,67 & & & \\
\hline Matrimonio $\geq 20$ años & & 4,08 & & & 3,26 & & & \\
\hline Pareja con $\mathrm{DE}^{\star}$ & & 24,35 & & & & & & \\
\hline Problemas financieros & & & 8,34 & & & & & \\
\hline Enfermedad crónica & & & 23,96 & & 8,52 & & & \\
\hline Menopausia & & & 2,26 & & 6,64 & & & \\
\hline $\begin{array}{l}\text { Uso de anticoncep- } \\
\text { tivo }\end{array}$ & & & 1,74 & & 0,68 & & & \\
\hline $\begin{array}{l}\text { Mayor nivel de esco- } \\
\text { laridad }\end{array}$ & & & & 3,84 & & & 1,86 & \\
\hline Historia de aborto & & & & 1,89 & & & & \\
\hline$>40$ años & & & & & 4,14 & 11,7 & 2,00 & \\
\hline Esposo $>47$ a & & & & & 4,29 & & & \\
\hline$>5$ partos & & & & & & & 0,26 & \\
\hline $\begin{array}{l}\text { Ciclo menstrual } \\
\text { anormal }\end{array}$ & & & & & & & 1,38 & \\
\hline Alcohol $^{1}$ & & & & & & & & 2,00 \\
\hline
\end{tabular}

*Disfunción eréctil, ${ }^{1}$ una botella por semana 
Tabla V

TASAS AJUSTADAS DE LOS FACTORES DE RIESGO DE DISFUNCIÓN SEXUAL FEMENINA

\begin{tabular}{|c|c|c|c|}
\hline Variables evaluadas & Estimador & Efecto & IC95\% \\
\hline \multicolumn{4}{|c|}{ Lo SS, 2014} \\
\hline Menos "juego previo" & OR & 2,29 & $(1,74-2,94)$ \\
\hline Menos frecuencia de relaciones sexuales & OR & 1,80 & $(1,43-2,29)$ \\
\hline Enfermedad médica & OR & 1,67 & $(1,12-2,48)$ \\
\hline Inicio unidireccional de la relación sexual & OR & 1,82 & $(1,51-2,20)$ \\
\hline Educación primaria & OR & 3,97 & $(1,15-13,87)$ \\
\hline \multicolumn{4}{|c|}{ Jaafarpour M, 2013} \\
\hline$\geq 40$ años & OR & 2,23 & $(1,12-2,68)$ \\
\hline Bajo nivel educativo & OR & 1,54 & $(1,09-2,13)$ \\
\hline$\leq 3$ relaciones sexuales por semana & OR & 1,85 & $(1,23-1,99)$ \\
\hline$\geq 3$ partos & OR & 1,48 & $(0,97-1,24)$ \\
\hline Esposo $\geq 40$ años de edad & OR & 2,11 & $(1,35-2,37)$ \\
\hline Matrimonio $\geq 10$ años & OR & 1,76 & $(1,04-1,97)$ \\
\hline Desempleada & OR & 1,34 & $(1,06-1,63)$ \\
\hline \multicolumn{4}{|c|}{ Choi H, 2014} \\
\hline$\geq 60$ años & OR & 2,09 & $(0,77-5,39)$ \\
\hline Actitud negativa sobre el sexo & OR & 1,26 & $(0,78-2,06)$ \\
\hline Fumar & OR & 2,76 & $(0,85-0,89)$ \\
\hline Mala comunicación con la pareja & OR & 2,11 & $(1,29-3,46)$ \\
\hline \multicolumn{4}{|c|}{ Grewal GS, 2014} \\
\hline Relaciones sexuales $\leq 2$ veces a la semana & OR & 6,74 & $(0,66-68,54)$ \\
\hline Matrimonio $\geq 20$ años & OR & 2,49 & $(0,43-14,32)$ \\
\hline Pareja con $\mathrm{DE}^{\star}$ & OR & 27,36 & $(4,71-159,08)$ \\
\hline \multicolumn{4}{|c|}{ Ibrahim ZM, 2013} \\
\hline$>44$ años & OR & 2,1 & $(1,6-3,5)$ \\
\hline Posmenopausia & OR & 4,2 & $(1,5-6,4)$ \\
\hline Matrimonio $\geq 10$ años & OR & 2,0 & $(1,2-4,3)$ \\
\hline Uso de terapia hormonal & OR & 0,1 & $(0,02-0,3)$ \\
\hline Circuncisión & OR & 6,5 & $(2,6-15,8)$ \\
\hline Pareja con $\mathrm{DE}^{\star}$ & OR & 4,4 & $(1.5-12,5)$ \\
\hline Pareja $>50$ años de edad & OR & 2,0 & $(1,1-8,3)$ \\
\hline \multicolumn{4}{|c|}{ Ghanbarzadeh N, 2013} \\
\hline Ama de casa & OR & 0,33 & $(0,12-0,95)$ \\
\hline Trabajar & OR & 0,08 & $(0,01-0,57)$ \\
\hline Relación satisfactoria con el esposo & OR & 17,16 & $(5,42-54,29)$ \\
\hline Vida sexual activa & OR & 6,66 & $(1,22-12,57)$ \\
\hline \multicolumn{4}{|c|}{ Zhang H, 2012} \\
\hline Universitarias & OR & 1,62 & $(0,90-2,88)$ \\
\hline Ama de casa & OR & 1,27 & $(0,95-1,70)$ \\
\hline Religiosas & OR & 1,07 & $(0,77-1,47)$ \\
\hline Pareja con $\mathrm{DE}^{\star}$ & OR & 2,44 & $(1,04-5,75)$ \\
\hline Actitud liberal sobre el sexo & OR & 0,63 & $(0,47-0,83)$ \\
\hline Historia de aborto & OR & 1,36 & $(0,96-1,93)$ \\
\hline
\end{tabular}


CONTINUACIÓN TABLA V

\begin{tabular}{|c|c|c|c|}
\hline Variables evaluadas & Estimador & Efecto & IC95\% \\
\hline \multicolumn{4}{|c|}{ Ishak IH, 2010} \\
\hline$>45$ años & OR & 1,49 & $(0,23-9,63)$ \\
\hline Esposo $>47$ años de edad & OR & 0,99 & $(0,15-6,52)$ \\
\hline Matrimonio $>20$ años & OR & 0,32 & $(0,06-1,74)$ \\
\hline Enfermedad medica de base & OR & 4,67 & $(1,55-14,03)$ \\
\hline Menopausia & OR & 3,32 & $(0,93-11,87)$ \\
\hline$\leq 3$ relaciones sexuales al mes & OR & 7,22 & $(2,17-24,00)$ \\
\hline \multicolumn{4}{|c|}{ Goshtasebi A, 2009} \\
\hline$\geq 46$ años & OR & 2,0 & $(1,05-3,8)$ \\
\hline Secundaria & OR & 2,61 & $(1,17-5,81)$ \\
\hline Trabajar & OR & 1,25 & $(0,75-2,09)$ \\
\hline Uso de anticonceptivos orales & OR & 0,64 & $(0,43-0,94)$ \\
\hline DIU & OR & 1,12 & $(0,53-2,39)$ \\
\hline \multicolumn{4}{|c|}{ Singh JC, 2009} \\
\hline Bajo nivel de escolaridad & OR & 1,2 & $(1,0-1,3)$ \\
\hline$>40$ años & OR & 8,2 & $(2,3-28,9)$ \\
\hline \multicolumn{4}{|c|}{ Chedraui P, 2009} \\
\hline$>46$ años & OR & 3,3 & $(1,6-6,8)$ \\
\hline Posmenopausia & OR & 2,8 & $(1,3-6,1)$ \\
\hline Pareja fiel & OR & 0,2 & $(0,1-0,4)$ \\
\hline Uso de terapia hormonal & OR & 0,4 & $(0,1-1,0)$ \\
\hline Esposo con DE & OR & 3,8 & $(1,3-10,9)$ \\
\hline Pareja mayor & OR & 2,0 & $(1,0-4,0)$ \\
\hline \multicolumn{4}{|c|}{ Song SH, 2008} \\
\hline Tener entre $20-29$ años & OR & 3,49 & $(1,48-8,41)$ \\
\hline Relaciones sexuales $5-7$ veces por mes & OR & 5,89 & $(1,70-20,37)$ \\
\hline Actitud negativa sobre el sexo & OR & 19,06 & $(5,67-64,06)$ \\
\hline No masturbarse & OR & 1,66 & $(1,04-2,64)$ \\
\hline Homosexualidad & OR & 16,65 & $(1,78-155,51)$ \\
\hline DIU & OR & 3,41 & $(0,99-11,76)$ \\
\hline \multicolumn{4}{|c|}{ Hayes RD, 2008} \\
\hline$>40$ años & OR & 0,4 & $(0,1-1,9)$ \\
\hline Postmenopausia & OR & 0,9 & $(0,1-5,6)$ \\
\hline Terapia hormonal & OR & 3,4 & $(0,5-20,7)$ \\
\hline Actualmente deprimida & OR & 3,1 & $(1,2-7,8)$ \\
\hline Educación adicional & OR & 0,8 & $(0,3-1,9)$ \\
\hline Buena comunicación con la pareja & OR & 0,2 & $(0,05-0,5)$ \\
\hline \multicolumn{4}{|c|}{ Amidu N, 2010} \\
\hline Alcohol $^{1}$ & OR & 2,0 & $(1,1-3,8)$ \\
\hline
\end{tabular}

DIU: Dispositivo intrauterino. ${ }^{1}$ Una botella por semana 
La menopausia demostró ser un factor de riesgo en todos los estudios en los cuales se evaluó esta variable $(18,19,20)$, excepto en el de Hayes y cols (11) (OR 0,9; IC95\% 0,1-5,6). Se encontró en dos de los estudios de la revisión $(13,20)$, que el uso de terapia de reemplazo hormonal constituye un factor protector para la presencia de DSF, pero Hayes y cols (11) no observó una asociación significativa (OR 3.4; IC95\% 0,5-20,7) (Tabla V).

En relación con la pareja se encontró que una mala comunicación o estar insatisfecha con el matrimonio, constituyen un factor de riesgo para desarrollar DSF $(1,16,19)$, mientras que presentar una buena comunicación con la pareja es un factor protector (OR 0,2; IC95\% 0,05-0,5) (11). La presencia de disfunción eréctil en la pareja según Grewal y cols (14) se constituye como unas de las variables más significativas de la revisión (OR 27,36; IC95\% 4,71-159,08), encontrándose también como significativa para la presencia de DSF en otros artículos en los cuales se evaluó $(14,19,20,22)$.

\section{DISCUSIÓN}

Los resultados de nuestro estudio muestran que la prevalencia de disfunción sexual femenina es de 5,5 a $73,2 \%$. Los factores de riesgo asociados significativamente a este problema fueron: baja frecuencia de relaciones sexuales, presencia de enfermedad médica de base, bajo nivel educativo, edad >40 años, matrimonio >10 años, desempleo o ser ama de casa, menopausia, mala comunicación con la pareja, presentar una pareja con disfunción eréctil. El rango de prevalencia encontrado en nuestro estudio acoge las prevalencias encontradas en la literatura donde se pueden encontrar prevalencias tan bajas y otras tan altas dependiendo del país donde se realizó el estudio, ya que ésta al igual que los factores de riesgo se ven influenciados por aspectos culturales de cada región. Ibrahim y cols (19) observaron que el $43 \%$ de las mujeres americanas menores de 59 años y el $52,8 \%$ de las mujeres egipcias presentan un tipo de disfunción sexual según el FSFI (19). En Asia, la incidencia de DSF es de $37,1 \%$ en mujeres jóvenes y de edad media casadas (22). En mujeres coreanas $<40$ años, se realizó una encuesta realizada en internet encontrándose que la prevalencia de disfunción sexual era de 43,1\% (24). En general, se observa una gran variabilidad en la prevalencia de disfunción sexual entre 5,5-73,2\%

El estudio de Grewal y cols (14) mostró la prevalencia más baja en toda la revisión, este autor desarrolló un estudio en mujeres que trabajaban en el área de la salud, lo que puede llevar a plantear varias hipótesis. Una de las posibles es que estas mujeres pudieron tener acceso a mayor información sobre la sexualidad, lo que las llevaría a tener una sexualidad plena y presentar una menor prevalencia; otra hipótesis es la baja frecuencia de relaciones sexuales no asociada con la DSF (OR 6,$74 ;$ IC95\% 0,66-68,54), lo cual puede influir en la disminución de la necesidad de tener relaciones sexuales, subestimando la prevalencia de disfunción sexual $(14,25)$.

La mayor prevalencia de DSF, se presentó en mujeres hindúes, debido posiblemente a la cultura y a la imposibilidad que tiene la mujer para tener acceso a una evaluación acerca de su sexualidad (15). Infortunadamente, el cuestionario FSFI no fue validado en esta población, lo que podría llevar a un posible sesgo de información de los resultados obtenidos.

El deseo fue el dominio con mayor prevalencia de disfunción, seguido del orgasmo. Este hallazgo es congruente con lo observado en la literatura. En diferentes estudios se recalca que los dominios más afectados en la sexualidad femenina son los dos mencionados anteriormente $(24,26,27)$. En el estudio de Grewal y cols (14), se encontró que las prevalencias más bajas en todos los dominios, excepto en el de deseo, la cual se encuentra en los demás estudios. Además, la asociación del bajo nivel educativo, la menor frecuencia de relaciones sexuales, la menopausia, una enfermedad de base, el estar deprimida y el consumo de alcohol con la presencia de algún tipo de disfunción sexual resultaron ser significativos, en concordancia con lo encontrado en la literatura. Es de resaltar que en 5 de los 9 artículos se observó un influencia de la edad y la presencia de DSF $(28,29)$.

Zhang y cols (22), evaluaron el efecto de la religión, pero no se encontró relación entre ser religiosa y presentar una disfunción sexual en esta población, lo mismo sucedió en el estudio de Ronsen y cols (30). Por el contrario, Stulhofer y cols (27), observaron que en mujeres croatas, la moralidad religiosa se asoció con la presencia de inhibición del deseo. Tener una pareja con disfunción sexual se evaluó en 3 de los 15 artículos revisados, encontrándose una asociación positiva significativa, siendo mayor el riesgo en el estudio de Grewal y cols (31).

La homosexualidad se encontró asociada positivamente a desarrollar algún tipo de disfunción sexual, como lo demuestra Song y cols (17). Sin embargo, en la revisión no se encontró estudios que evaluaran esta situación, por lo cual es necesario, la investigación y el análisis de disfunción sexual femenina en mujeres homosexuales.

Por otro lado, también se encontraron factores protectores frente a la disfunción sexual femenina como el uso de terapia hormonal, la actitud libre sobre el sexo, el tener una pareja fiel y la buena comunicación con la pareja (Tabla IV), lo que concuerdan con lo descrito en la literatura $(7,27)$.

Esta revisión posee las limitaciones que se pueden presentar en este tipo de artículos, que incluye 
la posibilidad de sesgo de publicación que se intentó reducir realizando una búsqueda exhaustiva y escogiendo los artículos que cumplieran con los criterios de inclusión y proporcionaran la información que permitieran su análisis. Esta búsqueda incluyó dos bases de datos principales, una en inglés y otra en español. Además, agregamos a la búsqueda electrónica una búsqueda en las referencias. La selección de estudios fue reproducible así como la asignación de criterios de calidad metodológica. La segunda limitación es que la revisión sistemática no cuantifica el impacto de los factores de riesgo unificados en la prevalencia de DSF, debido a la heterogeneidad de los factores evaluadas en cada artículo y a los modelos multivariados aplicados para dar respuesta a los objetivos de éstos. Los resultados del presente estudio permitirán orientar las intervenciones para reducir los factores de riesgo de las mujeres de desarrollar una disfunción sexual, dado que constituye la línea de base para la implementación de estrategias de intervención en el ámbito psicosocial.

\section{CONCLUSIÓN}

La disfunción sexual femenina posee una prevalencia muy alta entre la población femenina mundial. Existen factores individuales, culturales y sociales que influyen en la presencia de la DSF, su mejor entendimiento es vital para planificar estrategias innovadoras de los diferentes niveles de prevención, que reduzcan la progresión de la disfunción sexual y el impacto en la calidad de vida de las mujeres con esta condición. Es necesario el fortalecimiento de procesos investigativos en el área de disfunción sexual en Latinoamérica, que contribuya con una construcción de una política pública frente a la sexualidad femenina, que impactará en los determinantes sociales inductores para una equidad de género frente al abordaje de este tipo de enfermedades.

\section{REFERENCIAS}

1. Choi H, Kim JH, Park JY, Shim JS, Lee JG, Yoon HY, et al. Assessment of sexual dysfunction and determination of its risk factors in the Republic of Korea. Int $\mathrm{J}$ Gynaecol Obstet 2014;125(1):60-4.

2. Oksuz E, Malhan S. Prevalence and risk factors for female sexual dysfunction in Turkish women. J Urol 2006;175(2):654-8.

3. Basson R, Berman J, Burnett A, Derogatis L, Ferguson $\mathrm{D}$, Fourcroy $\mathrm{J}$, et al. Report of the international consensus development conference on female sexual dysfunction: definitions and classifications. J Urol 2000;163(3):888-93.

4. Wood A, Runciman R, Wylie KR, McManus R. An update on female sexual function and dysfunction in old age and its relevance to old age psychiatry. Aging Dis 2012;3(5):373-84.
5. Walton B, Thorton T. Female sexual dysfunction. Curr Womens Health Rep 2003;3(4):319-26.

6. Lo SST, Kok WM. Sexual behavior and symptoms among reproductive age Chinese women in Hong Kong. J Sex Med 2014;11(7):1749-56.

7. Laumann EO, Paik A, Rosen RC. Sexual Dysfunction in the United States. JAMA 1999;281(6):537-44.

8. Dennerstein L, Hayes RD. Confronting the challenges: epidemiological study of female sexual dysfunction and the menopause. J Sex Med 2005;2 Suppl 3:11832.

9. von Elma E, Douglas G, Altmanb DG, Eggera M, Pocockd SJ, Go PC, tzschee, Vandenbrouckef JP, en nombre de la IS. Declaración de la Iniciativa STROBE (Strengthening the Reporting of Observational studies in Epidemiology): directrices para la comunicación de estudios observacionales. Gac Sanit 2008;22(2):14450.

10. Rosen R, Brown C, Heiman J, Leiblum S, Ferguson D. The Female Sexual Function Index (FSFI): A Multidimensional Self-Report Instrument for the Assessent of Female Sexual Function. J Sex Marital Ther. 2000;26:191-208.

11. Hayes RD, Dennerstein L, Bennett CM, Sidat M, Gurrin LC, Fairley CK. Risk factors for female sexual dysfunction in the general population: exploring factors associated with low sexual function and sexual distress. J Sex Med 2008;5(7):1681-93.

12. Rust J, Golombok S. The GRISS: A psychometric instrument for the assessment of sexual dysfunction. Arch Sex Behav 1986;15(2):157-65.

13. Goshtasebi A, Vahdaninia M, Rahimi Foroshani A. Prevalence and potential risk factors of female sexual difficulties: an urban Iranian population-based study. J Sex Med 2009;6(11):2988-96.

14. Grewal GS, Gill JS, Sidi H, Gurpreet K, Jambunathan ST, Suffee NJ, et al. Prevalence and risk factors of female sexual dysfunction among healthcare personnel in Malaysia. Compr Psychiatry; 2014;55 Suppl 1:S1722.

15. Singh JC1, Tharyan P, Kekre NS, Singh G, Gopalakrishnan $G$. Prevalence and risk factors for female sexual dysfunction in women attending a medical clinic in south India. J Postgr Med 2009;55(2):113-20.

16. Jaafarpour M, Khani A, Khajavikhan J, Suhrabi Z. Female sexual dysfunction: prevalence and risk factors. J Clin Diagn Res 2013;7(12):2877-80.

17. Song SH, Jeon H, Kim SW, Paick J-S, Son H. The prevalence and risk factors of female sexual dysfunction in young korean women: an internet-based survey. J Sex Med 2008;5(7):1694-701.

18. Ishak IH, Low $\mathrm{W}-\mathrm{Y}$, Othman S. Prevalence, risk factors, and predictors of female sexual dysfunction in a primary care setting: a survey finding. $\mathrm{J}$ Sex Med 2010;7(9):3080-7.

19. Ibrahim ZM, Ahmed MR, Sayed Ahmed WA. Prevalence and risk factors for female sexual dysfunction among Egyptian women. Arch Gynecol Obstet 2013;287(6):1173-80.

20. Chedraui P, Perez-Lopez FR, San Miguel G, Avila C. Assessment of sexuality among middle-aged women using the Female Sexual Function Index. Climacteric 2009;12(3):213-21.

21. Elnashar AM, El-Dien Ibrahim M, El-Desoky MM, Ali OM, El-Sayd Mohamed Hassan M. Female sexual 
dysfunction in Lower Egypt. BJOG 2007;114(2):2016.

22. Zhang $\mathrm{H}$, Yip PSF. Female sexual dysfunction among young and middle-aged women in Hong Kong: prevalence and risk factors. J Sex Med 2012;9(11):2911-8.

23. Ghanbarzadeh N, Nadjafi-Semnani M, Ghanbarzadeh $M-R$, Nadjfai-Semnani A, Nadjfai-Semnani F. Female sexual dysfunction in Iran: study of prevalence and risk factors. Arch Gynecol Obstet 2013;287(3):533-9.

24. Sang Hoon Song JSP, Hwancheol S. The Prevalence and Risk Factors of Female Sexual Dysfunction in Young Korean Women: An Internet-Based Survey. J Sex Med. 2008;5(7):1694-701.

25. Edward O, Laumann P. Sexual Dysfunction in the United States Prevalence and Predictors. JAMA 1999;281(6):537-44.

26. Safarinejad MR. Female sexual dysfunction in a population-based study in Iran: prevalence and associated risk factors. Int J Impot Res 2006;18(4):382-95.
27. Stulhofer AGI. Sexual problems of urban women in Croatia: prevalence and correlates in a community sample. Croat Med J 2005;46(1):45-51.

28. Cayan S, Akbay E, Bozlu M, Canpolat B, Acar D, Ulusoy $E$. The prevalence of female sexual dysfunction and potential risk factors that may impair sexual function in Turkish women. Urol Int 2004;72(1):52-7.

29. Shifren JL, Monz BU, Russo PA, Segreti A, Johannes CB. Sexual problems and distress in United States women: prevalence and correlates. Obs Gynecol 2008;112(5):970-8.

30. Rosen RC, Taylor JF, Leiblum SR, Bachmann GA. Prevalence of sexual dysfunction in women: results of a survey study of 329 women in an outpatient gynecological clinic. J Sex Marital Ther 1993;19(3):171-88.

31. Jahan MS, Billah SM, Furuya $H$, Watanabe T. Female sexual dysfunction: facts and factors among gynecology outpatients. J Obs Gynaecol Res 2012;38(1):32935. 